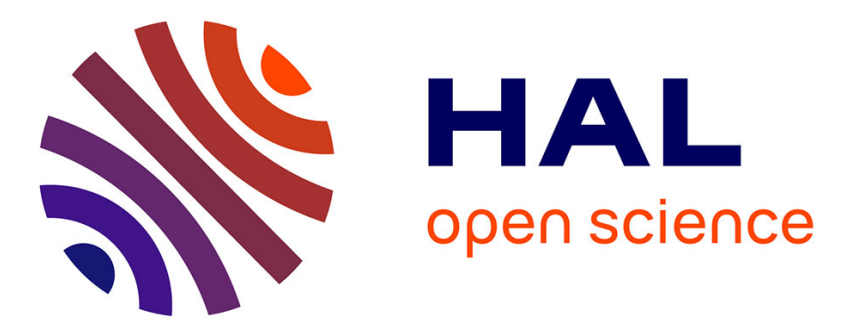

\title{
Assessment of Deterministic Shape Optimizations Within a Stochastic Framework for Supersonic Organic Rankine Cycle Nozzle Cascades
}

\author{
Alessandro Romei, Pietro Marco Congedo, Giacomo Persico
}

\section{To cite this version:}

Alessandro Romei, Pietro Marco Congedo, Giacomo Persico. Assessment of Deterministic Shape Optimizations Within a Stochastic Framework for Supersonic Organic Rankine Cycle Nozzle Cascades. Journal of Engineering for Gas Turbines and Power, 2019, 141 (7), 10.1115/1.4042925 . hal-03071659

\author{
HAL Id: hal-03071659 \\ https://hal.inria.fr/hal-03071659
}

Submitted on 16 Dec 2020

HAL is a multi-disciplinary open access archive for the deposit and dissemination of scientific research documents, whether they are published or not. The documents may come from teaching and research institutions in France or abroad, or from public or private research centers.
L'archive ouverte pluridisciplinaire HAL, est destinée au dépôt et à la diffusion de documents scientifiques de niveau recherche, publiés ou non, émanant des établissements d'enseignement et de recherche français ou étrangers, des laboratoires publics ou privés. 


\title{
Assessment of Deterministic Shape Optimizations
}

\author{
within a Stochastic Framework for
}

\section{Supersonic Organic Rankine Cycle Nozzle Cascades}

\author{
Alessandro Romei*1, Pietro Marco Congedo ${ }^{2}$, and Giacomo Persico ${ }^{1}$ \\ ${ }^{1}$ Laboratory of Fluid Machines (LFM), Energy Department - Politecnico di \\ Milano, Via Lambruschini 4, 20156 Milan, Italy. \\ ${ }^{2}$ DeFI Team (INRIA SIF, Ecole Polytechnique), CMAP Lab, 1 Rue \\ d'Estienne d'Orves, 91120 Palaiseau, France. \\ *Corresponding author: alessandro.romei@polimi.it
}

This is a preprint of the following article: Alessandro Romei, Pietro Marco Congedo and Giacomo Persico, "Assessment of Deterministic Shape Optimizations within a Stochastic Framework for Supersonic Organic Rankine Cycle Nozzle Cascades", J. Eng. Gas Turbines Power, vol. 141(7), pp. 1-11, 2019.

The published article may differ from this preprint, and is available at the following DOI: $10.1115 / 1.4042925$ 


\section{Abstract}

The design of converging-diverging blades for Organic Rankine Cycle applications widely relies on automated shape-optimization processes. As a result, the optimization produces an adapted-nozzle cascade at the design conditions. However, only few works accounts for the uncertainties in those conditions and their consequences on cascade performance. The proposed solution, i.e. including uncertainties within the optimization routine, demands an overall huge computational cost to estimate the target output statistic at each iteration of the optimization algorithm. With the aim of understanding if this computational cost is avoidable, we study the impact of uncertainties in the design conditions on the robustness of deterministically optimized profiles.

Several optimized blades, obtained with different objective functions, constraints and design variables, are considered in the present numerical analysis, which employs a turbulent flow solver and a state-of-the-art uncertainty-quantification method. By including measured field variations in the formulation of the uncertainty-quantification problem, we show that a deterministic shape optimization already improves the robustness of the profile with respect to the baseline configuration. Guidelines about objective functions and blade parametrization for deterministic optimizations are also provided. Finally, a novel methodology to estimate the mass-flow-rate probability density function for choked supersonic turbines is proposed, along with a robust reformulation of the constraint problem without increasing the computational cost. 


\section{INTRODUCTION}

Nowadays, Organic Rankine Cycle (ORC) power systems are a cost-competitive solution to exploit heat sources at medium-low enthalpy level [1]. The success of this technology mainly relies on a suitable design of its turboexpander [2], where the working fluid is a heavy and complex organic compound. These fluids feature low enthalpy drop combined with comparatively high expansion ratio, large volumetric variation, and low speed of sound. These specific features led designers to focus on compact axial [3, 4] or radial-inflow [5, 6] configurations characterized by one or a few stages. In case of multi-stage axial turbines, the optimization drives the design towards a first supersonic stator, which processes a significant fraction of the expansion ratio [7]. In case of radial-inflow turbines, the difficulty to arrange a multi-stage architecture demands the entire expansion process to be handled in a single stage, thus splitting the expansion in two cascades only. This layout induces severe supersonic flows in the radial-inflow nozzle $[8,9]$, posing the same design issues also characterizing the first-stage axial stator. Moreover, these cascades work in close proximity to the saturation curve, facing severe non-ideal gas effects in the expansion process.

Supersonic flows combined with non-ideal gas effects result in a very challenging aerodynamic design process of the blading. Novel semi-analytical design guidelines have been recently proposed $[10,11]$ to define the blade channel shapes of such nozzles in a costeffective way. However, the design of these machines can greatly benefit from the application of automated shape-optimization methods $[12,13,14,15]$. These design methods lead to converging-diverging cascades which resemble an adapted nozzle in design conditions. However, the introduction of uncertainties in the turbine operating conditions may unpredictably change its performance due to the complex interactions between non-ideal gas effects and supersonic flow regime. Therefore, a robust design, i.e. a design which is less sensitive to the system uncertainties, has to be devised [16] to ensure competitive performance in real 
field operations. This problem was already tackled by some authors: a first attempt at optimization under uncertainties for dense-gas flow applications was carried out by considering an isolated airfoil [17] and later a transonic cascade [18]. Recently, a sensitivity analysis of upstream flow quantities and geometrical uncertainties was carried out [19], followed by a multi-objective robust optimization [20].

Gathering information from all these previous studies, a discrepancy about which parameters should be taken as uncertain emerges, in the absence of a systematic study about ORC blades operating under uncertainties. Moreover, even though attempts of robust optimizations were carried out, no evidence is presently available on the lack of robustness of optimal configurations obtained by a deterministic optimization process. The latter is a necessary condition to justify the (very high) computational effort required by robust optimization techniques.

This work aims at providing such evidences, by performing an uncertainty-quantification analysis of several supersonic nozzle cascades for ORC applications including realistic operating uncertainties. Five blade configurations are investigated, by combining different objective functions, constraints and design variables in the deterministic-optimization set up. A turbulent compressible flow solver is employed along with a state-of-the-art uncertaintyquantification (UQ) method, based on the Polynomial Chaos-Kriging surrogate approximation. Flow (inlet total temperature and pressure, flow direction and outlet static pressure) and turbulent (turbulent intensity and eddy viscosity ratio) boundary conditions are taken as uncertain parameters, referring to real field variability [21].

The paper is structured as follows: Section 2 is devoted to the definition of the problem, deriving the optimized cascades and the corresponding uncertainties for the design conditions. Then, in Section 3 the numerical tools, i.e. the flow solver and the uncertaintyquantification strategy, are illustrated. A systematic study for the statistic and the grid convergence is also reported to verify the uncertainty-quantification set up. In Section 4, 
after a dedicated discussion on the meaning of constraint fulfilment within a probabilistic framework, a simplified model to predict mass-flow-rate probability density functions (PDFs) is proposed for choked supersonic turbines. Finally, cascade performance, which satisfy the aforementioned constraint on the mass-flow rate, are discussed from both a physical and a quantitative standpoint. This analysis is instrumental to derive guidelines which allow a proper tuning of deterministic shape optimizations to achieve robust performance of the resulting optimized blades. In the last section, some conclusions about the need of a robust optimization procedure are drawn and future works are prospected.

\section{PROBLEM DEFINITION}

\subsection{Blade configurations}

The analysis concerns a supersonic axial-flow turbine stator featuring converging-diverging blades [3]. The working fluid is MDM $\left(\mathrm{C}_{8} \mathrm{H}_{24} \mathrm{O}_{2} \mathrm{Si}_{3}\right.$, octamethyltrisiloxane), which exhibits relevant non-ideal effects in the considered operating range. From a deterministic standpoint, the flow is expanded from superheated conditions $\left(\mathrm{T}_{\mathrm{T} 0}=543.15 \mathrm{~K}\right.$ and $\mathrm{P}_{\mathrm{T} 0}=8$ bar $)$ with an expansion ratio of $7.5\left(\mathrm{P}_{1}=1.072 \mathrm{bar}\right)$.

The original cascade, referred to as baseline in the following and shown in Figure 1, was the subject of several optimization trials in recent years $[13,14,15]$. In the present work, the baseline as well as the following optimized blades are studied from a UQ perspective.

All optimal blades are obtained by applying the tool FORMA (Fluid-dynamic OptimizeR of turboMachinery Aerofoils) [14, 22], applying different objective functions, constraints, and design variables. In the context of FORMA, the baseline blade is parametrized by resorting to a B-Spline technique. A non-uniform control-point (CP) distribution is prescribed for the blade parametrization, locally increasing the number of CPs where the blade curvature is high. The cascade flow field suggests that the most critical region for the performance is 
downstream of the throat section, due to the onset of shock waves at the trailing edge and on the rear suction side of the blade. An analysis of variance [23] devoted to the evaluation of the impact of control point positions on the cascade performance confirmed quantitatively this deduction, attributing a higher relative weight to the CPs placed on the rear suction side. CFD simulations are run using a high-fidelity flow model and a surrogate-based evolutionary strategy is employed to reduce the computational cost of the optimization.

With reference to the design space reported in Figure 1(a), which features 13 movable CPs in the rear part of the blade, two objective functions are considered, namely the minimization of the entropy production across the cascade (labelled hereinafter DS) and the minimization of the standard deviation of azimuthal pressure distribution evaluated at half of the axial chord downstream of the trailing edge (labelled hereinafter DP):

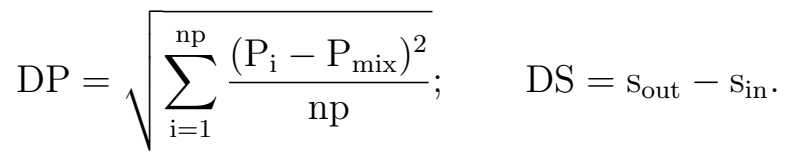

$\mathrm{np}$ is the number of computational points along the azimuthal direction on the downstream traverse, where the pressure $\mathrm{P}_{\mathrm{i}}$ is computed. $\mathrm{P}_{\text {mix }}$ is the mixed flow pressure. $\mathrm{s}_{\text {out }}$ and $s_{\text {in }}$ are the mass-averaged specific entropy at the outlet and inlet domain respectively.

These definitions are motivated by the interest in maximizing the stator aerodynamic performance and/or the uniformity of the flow at the inlet of the subsequent rotor. For the two objective functions, both unconstrained and constrained optimizations are performed. The constrained optimization exploits a penalty formulation, where the difference between the actual constraint value and the specified threshold is added to the objective function after multiplication by a penalty coefficient, which establishes both the quantitative homogeneity with the objective function and the weight of the constraint. In this work, the mass-flow rate is constrained to remain within $\pm 1 \%$ of the baseline value.

The combination of objective functions (DP, DS) and constraints (CNS) identifies 4 
optimal blades, namely 13CP-DP, 13CP-DS, 13CP-CNS-DP, and 13CP-CNS-DS. For one of these cases, i.e. the constrained minimization of the entropy generation, a comparative optimization is performed in a reduced design space, reported in Figure 1(b), featuring 7 movable Control Points placed on the rear suction side only (7CP-CNS-DS).

The resulting five optimal individuals are illustrated in Figure 2. Geometrical differences among optimal blades, which can be better appreciated in the zoom of Figure 2, mainly involve the trailing edge and the pressure side as a consequence of different problem formulations. On the other hand, a common element distinguishes the optimal blades with respect to the baseline. Indeed, optimal blades feature a higher curvature in the diverging channel and an almost straight profile downstream of the cascade opening. To proper appreciate the flow configuration in this class of cascades and the impact of the optimization on the cascade aerodynamics, Figure 3 shows the Mach number distributions for the baseline configuration and for the 13CP-DS profile, taken as representative of the optimal cascades. The baseline cascade features a strong shock, clearly visible in Figure 3(a), generated by the recompression occurring in the rear suction side, and due to the local surface curvature. As a result of the optimization, the main shock observed in the baseline cascade is almost eliminated, thus significantly improving the aerodynamic performance of optimal cascades. Only the fish-tail shock, which stems from the finite thickness of the trailing edge, is reflected on the rear suction side of the adjacent blade, as illustrated by the representative optimized profile 13CP-DS in Figure 3(b). The optimized cascades feature a more uniform flow field downstream of the throat, also evidenced by an almost straight path of the wake, conversely to the waving character induced by the main shock in the baseline layout.

The corresponding performance, evaluated with the same computational flow model used for the UQ analysis and presented in Section 3, are listed in Table 1. The table reports, along with the evaluation of the objective functions and of the constraint, the total-pressure 
loss coefficient, which quantifies the cascade-aerodynamic performance:

$$
\mathrm{Y}=\frac{\mathrm{P}_{\mathrm{T}, \text { in }}-\mathrm{P}_{\mathrm{T}, \text { out }}}{\mathrm{P}_{\mathrm{T}, \text { in }}-\mathrm{P}_{\text {out }}}
$$

\subsection{Uncertainties Definition}

In the present study, we formulate the UQ problem by identifying two groups of uncertainties: physically-relevant and physically-unknown uncertainties.

Regarding the first category, we neglect uncertainties in the thermodynamic model, because previous works proved that they affect cascade performance in a negligible way compared to the operating uncertainties [17, 18]. These latter, i.e. uncertainties in the NavierStokes boundary conditions, are extrapolated by referring to field-property variations in an existing ORC power plant [21]. Despite a different working fluid and turbine layout with respect to the present case, those data are the only available in literature to the authors' best knowledge, and give a clear indication about their order of magnitude. Upstream-quantity uncertainties are taken as the maximum range of variation measured in the power plant. As far as the downstream static pressure is concerned, only condensation-temperature measurements are reported. Therefore, the latter are propagated via a Monte Carlo simulation to derive the corresponding back-pressure variation. This operation is performed by considering the thermodynamic properties of MDM rather than the organic fluid employed in the power plant.

Unfortunately, there are no similar indications in literature to derive plausible uncertainties also for turbulence and for flow direction. The latter is arbitrarily assumed to vary within $\pm 5 \mathrm{deg}$. Due to the lack of information about turbulence measurements in ORC power plants, we assign comparatively large uncertainties in the turbulence intensity and eddy viscosity ratio.

Finally, uniform distributions are assumed for all uncertainties, since no evidence about 
a specific prior distribution is provided in literature. Lower and upper values are taken symmetric with respect to the mean value (corresponding to the deterministic values reported in Section 2). Table 2 reports a complete summary of the uncertainties and related ranges employed in the following UQ studies.

\section{$3 \quad$ NUMERICAL TOOLS}

In this section, the flow solver and the thermodynamic model are described. For a fair comparison, the same flow model is also used within the previous optimization processes. Then, the UQ method is discussed along with its verification for the statistic and the grid convergence.

\subsection{Flow Model}

Numerical simulations are performed with ANSYS-CFX 18.1 ${ }^{\circledR}$, setting total quantities $\left(\mathrm{P}_{\mathrm{T} 0}\right.$, $\left.\mathrm{T}_{\mathrm{T} 0}\right)$ and flow angle $\left(\alpha_{0}\right)$ as inlet boundary conditions. The outlet boundary condition is imposed as static pressure $\left(\mathrm{P}_{1}\right)$, formulated as average static pressure and accepting an oscillation of $5 \%$ around the mean value. Moreover, the outlet domain is placed at four axial chords downstream of the trailing edge to avoid spurious pressure-wave reflection. As only the blade-profile aerodynamics are of interest, the quasi-3D blade-to-blade flow model is applied, considering a stream-tube of constant cross-section.

The turbulence model is $\mathrm{k}-\omega \mathrm{SST}$, whose boundary conditions are set as Turbulence Intensity TI and eddy viscosity ratio $\mu_{\mathrm{t}} / \mu$. The deterministic performance reported in Table 1 are obtained by using design flow conditions as presented in Section 2, purely axial flow and turbulent boundary conditions equal to $\mathrm{TI}=5 \%$ and $\mu_{\mathrm{t}} / \mu=50$. Meshes are generated with ANSYS-TURBOGRID ${ }^{\circledR}$, by imposing a proper cell clustering near blade profile to ensure $\mathrm{y}^{+}<1$ in every condition. The implemented thermodynamic model is the Span-Wagner 
equation of state as formulated in [24], by a Look-up-Table (LuT) approach. Thermodynamic tables are built by referring to the NIST REFPROP ${ }^{\circledR}[25]$ database, which also makes available dedicated correlations for transport properties. High-resolution schemes are employed in the discretization of both flow and turbulence equations. A central difference scheme is instead adopted for the diffusive flux. The computational flow solver, in terms of physical model, discretization schemes and mesh settings, was previously validated against experimental data [26].

Furthermore, a dedicated grid convergence assessment for the baseline cascade was performed at the beginning of this study and its main results are proposed in Figure 4 for the two objective functions discussed in this work (see Equation 1). The mesh composed by 200k cells shows the best compromise between computational cost and accuracy ( $0.4 \%$ and $1.7 \%$ of deviations for pressure-based and entropy-based objective functions, respectively, with respect to the finest mesh). Based on this evidence, all deterministic results discussed in this work are computed with the 200k mesh.

\subsection{Uncertainty Quantification Method}

The UQ analyses are carried out with a recently-proposed surrogate strategy called Polynomial Chaos-Kriging (PCK). The combination of these two surrogate models results in a metamodelling technique which is more efficient than Polynomial Chaos and Kriging taken separately [27]. First, a Latin Hypercube Sampling (LHS) algorithm is used to generate a Design of Experiments (DoE), where the surrogate model is trained. The number of samples to have a proper representation of the CFD response is discussed in the next subsection. The PCK strategy couples the advantages of a Kriging, which fits the observed value, with a Polynomial Chaos expansion, which instead better approximates the global trend of the function. As a matter of fact, it is a universal Kriging model, i.e. a stationary Gaussian process, whose mean trend is represented by a weighted sum of multivariate polynomials 
orthonormal with respect to the input distribution. The model can be expressed by the following equation:

$$
\mathrm{QoI}=\sum_{|\mathbf{i}|=0}^{\mathrm{N}} \beta_{\mathrm{i}} \Psi_{\mathrm{i}}(\mathbf{X})+\sigma^{2} \mathrm{Z}(\mathbf{X})
$$

where $\mathbf{X}=\left[\mathrm{T}_{\mathrm{T} 0}, \mathrm{P}_{\mathrm{T} 0}, \mathrm{P}_{1}, \mathrm{TI}, \alpha_{0}, \mu_{\mathrm{t}} / \mu\right]$ is the vector containing the uncertain variables, while QoI represents the Quantity of Interest, namely the mass-flow rate (m), the standard deviation of azimuthal pressure distribution (DP), the entropy production (DS) and the total-pressure loss coefficient (Y). We built one surrogate model for each QoI considered in the present study. In general, the first term of Eq. 3 is the model trend given by Polynomial Chaos expansion, while $\mathbf{Z}(\mathbf{X})$ is the zero mean, unit variance, stationary Gaussian Process, with covariance $\mathrm{R}\left(\mathbf{x}, \mathbf{x}^{\prime}, \boldsymbol{\theta}\right)$. The latter is chosen as a Matérn function with $\nu=5 / 2$ [28]. The optimal set of orthonormal polynomials are determined by a sparse Polynomial Chaos expansion based on Least Angle Regression Selection (LARS) [29]. Finally, the Kriging surrogate is calibrated by solving the maximum likelihood problem via genetic algorithms, thus determining model parameters $\left(\boldsymbol{\theta}, \beta_{i}, \sigma\right)$.

Once the PCK model is generated, it can be exploited to perform a global sensitivity analysis. Under this perspective, we computed total Sobol indices $\mathrm{S}_{\mathrm{i}}^{\mathrm{T}}(\mathrm{QoI})$, representing the total relative contribution of each variable to the QoI. The definition of Sobol indices stems from the existence of a unique Sobol functional decomposition. If the QoI is square-integrable, i.e. $\mathrm{QoI} \in \mathrm{L}^{2}(\mathbf{X}, \mathrm{P}(\mathbf{X}))$, also the variance can be decomposed into different contributions attributable to each input or any their interactions. For example, the first order Sobol index is by definition the ratio between the contribution given by the i-th variable to the variance and the total variance:

$$
\mathrm{S}_{\mathrm{i}}^{\mathrm{I}}=\frac{\operatorname{Var}\left[\mathrm{E}\left[\mathrm{QoI} \mid \mathbf{X}_{\mathrm{i}}\right]\right]}{\operatorname{Var}[\mathrm{QoI}]}
$$


To clarify the meaning of this statistical index, a first order index $\mathrm{S}_{\mathrm{i}}^{\mathrm{I}}(\mathrm{Q} \mathrm{OI})$ represents the contribution of each variable taken alone, the second order index $S_{\mathrm{ij}}^{\mathrm{II}}(\mathrm{QoI})$ accounts for the cross interaction of the variable $i$ and $j$ which cannot be decomposed into a single contribution of each of these variables, and so on for higher order indices. The analytical expressions for higher order indices recall the one provided in Eq. 4 for the first order index. The summation of all Sobol's indices involving the i-th variable gives the total Sobol index $\mathrm{S}_{\mathrm{i}}^{\mathrm{T}}(\mathrm{QoI})$, which is estimated by computing its Janon estimator [30] via a Monte-Carlo sampling. The whole surrogate strategy and the sensitivity analysis are implemented within UQLab framework [31].

Finally, the surrogate response is also used to generate the PDF of the QoIs. This is again done by a Monte-Carlo sampling evaluated through the PCK surrogate. Output values are collected in histograms on which a Kernel Density Estimation (KDE) is applied. The bandwidth of the kernel density estimator is chosen according to Silverman's rule [32], which may give inaccurate results when multi-modal distributions are present. For this reason, we check from the histograms that the empirical distributions are not multi-modal, otherwise a manual tuning of the bandwidth is operated.

\subsection{Statistic and Grid Convergence}

To properly set up the UQ method, an assessment of the grid and of the statistic convergence is conducted, in terms of (i) grid refinement and (ii) DoE size, on which the surrogate PCK is trained, respectively. Starting with the mesh refinement, so far all previous UQ analyses focused on the grid convergence from a deterministic perspective, assuming that the discretization error is preserved when moving to a stochastic level. However, this assumption is not necessarily valid, because a mesh, which is judged sufficiently accurate for deterministic purposes, might instead alter the hierarchy of uncertainties or the PDF shape when uncertainties are propagated through the numerical solver. Therefore, the assessment of the 
grid impact on the stochastic response is necessary to confirm the reliability of the method. Figure 5 reports the three grids tested in the present study, consisting in $50 \mathrm{k}, 200 \mathrm{k}$ and $500 \mathrm{k}$ cells respectively. Note also that even the coarsest mesh features a proper cell distribution near the blade wall to ensure $\mathrm{y}^{+}<1$ during the UQ assessment. Figure 6 reports total Sobol indices and PDFs of the mass-flow rate $(a-b)$ and of the entropy production $(c-d)$. The verification is performed on the representative optimized profile 13CP-DP. The DoE size is identical for the three meshes in order to isolate the geometrical-discretization error on the overall result. The coarsest grid is suitable for the mass-flow-rate characterization, which presents negligible variations by increasing the mesh size both in the sensitivity indices, Figure 6(a), and in the PDF shapes, Figure 6(b). Of course, this evidence has a clear physical interpretation. As the supersonic turbine is choked, the mesh size affects the mass-flow rate only until the throat section: imposing a $\mathrm{y}^{+}<1$ at the blade wall, the mesh is locally fine in the bladed channel, exactly where it is important for the mass-flow-rate computation. A different trend is observed if the entropy production is concerned. For this QoI, the coarsest grid entails an overestimation of the total temperature impact on the entropy production, see Figure $6(c)$. Conversely, the 200k mesh exhibits trends which are comparable to the finest one. Moreover, although PDF shapes are comparable, see Figure $6(d)$, the one generated with 50k mesh reports a mean entropy production which is higher than the corresponding finest value of around $7 \%$. The same quantity, but evaluated with $200 \mathrm{k}$, shows a deviation of around $1.5 \%$ with respect to the finest value, which is in a fair agreement with the gridconvergence result for the deterministic computations. Therefore, the intermediate mesh represents the best trade-off between computational cost and accuracy.

Finally, two DoE sizes, composed by 100 and 200 samples, respectively, are compared with reference to the intermediate mesh. The comparison is reported in Figure 6. Again, massflow rate does not evidence any difference neither in the sensitivity indices nor in the PDF shapes. On the other hand, entropy production presents a small deviation by increasing the 
number of samples, also revealing a slightly different interaction among input uncertainties. However, the difference is small (lower than $0.5 \%$ in the mean values) and does not alter the uncertainty hierarchy.

Based on these results, the intermediate mesh with 200k cells and the smallest DoE size with 100 samples are retained in the following calculations.

\section{RESULTS}

In this section, a possible interpretation of constraint fulfilment under uncertainties in terms of mass-flow rate is proposed, also providing a straightforward method for its prediction in chocked supersonic turbines. This analysis is instrumental to understand whether an optimal blades is worth to be analysed in subsequent sections. On the one hand, nozzle cascades, which do not satisfy the mass-flow constraint, are considered not technologically relevant, therefore they are not included in further comparisons. On the other hand, technologically-relevant blades are analysed in terms of cascade performance and flow-field uniformity, bearing in mind the deterministic-optimization problems to which they belong. These information are inferred to establish the optimal set up in terms of design space, constraint and objective function formulation to achieve the best performance when the blade is working under uncertainties.

\subsection{Robust mass-flow-rate constraint formulation}

The formulation of a shape-optimization problem for supersonic turbines in power systems demands the constraint of mass flow rate to match the power target. For subsonic turbines, due to the strong relationship between mass-flow rate and velocity triangles, the optimization problem can be reformulated by fixing the outlet flow angle. However, this is not possible when dealing with choked supersonic turbines, as the mass-flow rate is decoupled from what 
is occurring downstream. A direct constraint on this quantity is therefore necessary to find optimal blades which are also technologically relevant. The most straightforward way to deal with constrained-optimization problem is to apply a penalty formulation, i.e. a penalty is added to the objective function penalizing those individuals which do not satisfy the constraint. When dealing with non-linear problems, considerable improvements of the objective function can be obtained by accepting a small degree of unfulfillment. As far as the mass-flow rate is concerned, up to a certain value, a small variation of the mass-flow rate discharged by the 2D profile can be compensated by a variation in the blade height, justifying a small discrepancy in the mass-flow rate value with respect to the baseline. However, the problem becomes more complex when uncertainties are introduced, as a constraint may be satisfied or not according to a certain probability. In this work, we want to provide evidence about the main quantities affecting the mass-flow rate for supersonic nozzle cascades to understand whether the mass-flow rate should be included in a wider framework of robust optimization.

Total Sobol indices and PDFs for the five optimized blades are reported in Figure 7. As previously highlighted, not all blades are found via a constrained-optimization problem; examples are 13CP-DP and 13CP-DS. In the stochastic framework, a blade which does not deterministically fulfill a constraint might satisfy the same constraint with some probability. The first thing to notice by inspecting total Sobol indices, see Figure 7(a), is that, for all blades, only upstream total quantities influence the mass-flow rate. Among them, the total pressure always plays a major role with respect to the total temperature $\left(\mathrm{S}_{\mathrm{P}_{\mathrm{T} 0}}^{\mathrm{T}}(\dot{\mathrm{m}}) \approx 0.85\right.$ vs $\mathrm{S}_{\mathrm{T}_{\mathrm{T} 0}}^{\mathrm{T}}(\dot{\mathrm{m}}) \approx 0.15$, they add up to 1 , no cross-interaction is present). Coherently with what is expected for a choked supersonic turbine, the downstream static pressure does not have any effect on the mass-flow rate. This is true as long as the variation in static pressure is large enough so that the blade is always in choked conditions. Moreover, neither turbulent boundary conditions nor incidence angle have any influence. Once we have understood 
which elements contribute to the mass-flow rate, we still need to figure out: (i) how large the mass-flow-rate uncertainty is; (ii) to what extent the constraint is fulfilled.

Mass-flow-rate PDFs along with the prescribed acceptable range for the deterministic optimizations $( \pm 1 \%)$ are reported in Figure $7(b)$. The probability of the constraint being satisfied is proportional to the overlapped area between the target PDF and the deterministic threshold. It follows that blades optimized by direct imposition of the constraint satisfy the constraint with a large probability (> 70\%), but still PDF tails fall out the prescribed range.

As the PDF support is wider than the acceptable tolerance on the constraint, the robust fulfilment of the constraint cannot be ensured with $100 \%$ probability without reducing the input uncertainties. On the other hand, blades optimized without any direct constraint exhibit different behaviours: 13CP-DP satisfies the constraint with a probability of nearly 9\% while 13CP-DS never satisfies it. Therefore, a deterministic imposition of the constraint drives the optimization towards a more robust fulfilment, i.e. the constraint is satisfied with a larger probability. However, although the constraint is never satisfied with $100 \%$ probability, the computed mass-flow-rate uncertainties are not large enough to make undistinguishable profiles obtained with and without constrained-optimization problems.

The knowledge of a simple way to reconstruct the PDF without resorting to an expensive UQ algorithm can be used to formulate an alternative optimization problem, which enforces the constraint-fulfilment robustness at the same computational cost. Indeed, the similar shape of the PDFs along with nearly identical total Sobol indices suggest a pattern that can be deduced a priori. The mass-flow rate can be easily computed by referring to the throat section $(M=1)$, assuming isentropic flow in the convergent part of the blade along with the conservation of the total enthalpy:

$$
\dot{\mathrm{m}}=\rho_{\mathrm{t}} \mathrm{A}_{\mathrm{t}} \mathrm{c}_{\mathrm{t}}
$$


where the subscript $t$ indicates the throat section, $\rho$ is the density, $A$ is the cross-sectional area and $c$ is the speed of sound. Upon examination of Equation 5, the density and the speed of sound in the throat section are affected by a variation in the upstream total quantities, while the cross-sectional area is constant. The sensitivity analysis demonstrated no crosscorrelation between the two total quantities, so their contribution to the mass-flow rate can be regarded separately. The impact of the total-pressure variation on the speed of sound is practically negligible in these thermodynamic conditions, hence an increase in the total pressure produces an increase of the mass-flow rate as a consequence of the density rise. Since the relationship between pressure and density (at fixed temperature) is practically linear in those range of variations, a uniform pressure distribution will directly translate into an uniform mass-flow-rate distribution. A careful inspection of the mass-flow-rate PDFs, see Figure $7(b)$, reveals a flat central region which actually resembles an uniform distribution. Moreover, this region is predominant in the definition of the whole probability function, in agreement with the higher contribution of the total pressure to the mass-flow-rate uncertainty unveiled by the sensitivity analysis. By process of elimination, the total-temperature variation should be responsible for the PDF tails, which differentiate the mass-flow-rate PDF from an uniform distribution. Indeed, the total temperature affects both density and speed of sound, but in an opposite way: in these thermodynamic conditions, as the temperature increases, the density decreases but the speed of sound increases. The competitive effects of the total temperature in the mass-flow rate can explain the stiffer response in the resulting PDFs. The overall symmetry of the examined PDF with respect to the mean value can be a consequence of the symmetric interval chosen for the input uncertainties.

Gathering these information, a simple methodology to predict the mass-flow-rate PDF is proposed. With reference to the extreme values of total pressure and total temperature, namely $\mathrm{P}_{\mathrm{T} 0 \text {,min }}-\mathrm{P}_{\mathrm{T} 0 \text {, max }}$ and $\mathrm{T}_{\mathrm{T} 0, \min }-\mathrm{T}_{\mathrm{T} 0 \text {,max }}$, respectively, let us consider all the possible permutations of upstream total states $\left(\mathrm{P}_{\mathrm{T} 0}, \mathrm{~T}_{\mathrm{T} 0}\right)$. Once the upstream total state is identified, 
the mass-flow rate can be computed by solving the 1D problem in the Equation 5 . Then, the mass-flow-rate value has to be properly collocated, according to the related upstream state, to form the trapeze as in Figure $8(a)$. Finally, the trapeze height has to be computed by imposing the total area equal to 1 , thus generating a consistent mass-flow-rate PDF.

In Figure $8(b)$ the PDF obtained through the UQ model and the 1D-based model are compared. The proposed model perfectly predicts the mass-flow-rate PDF, showing practically no differences between the analytical and the numerical probability functions. This simplified model to estimate the mass-flow-rate PDF can be combined with deterministic optimization to account for the inherent constraint violations. For example, the penalty to the objective function can be applied whenever

$$
\operatorname{Pr}\left(0.99 \dot{\mathrm{m}}_{\text {base }}<\dot{\mathrm{m}}<1.01 \dot{\mathrm{m}}_{\text {base }}\right)<\beta
$$

where $\beta$ is the acceptable probability of the mass-flow rate deviating $\pm 1 \%$ from the baseline value, rather than the pure deterministic formulation:

$$
0.99 \dot{\mathrm{m}}_{\text {base }}>\dot{\mathrm{m}}>1.01 \dot{\mathrm{m}}_{\text {base }}
$$

In this way, the robust fulfilment of the constraint is enforced without an explicit and time-consuming uncertainty propagation. In principle, the robust mass-flow-rate constraint can be formulated for any choked supersonic turbine subjected to uniform uncertainties in the inlet total pressure and temperature.

\subsection{ANOVA of cascade-loss coefficient}

Before comparing the optimized blades which satisfy the mass-flow-rate constraint at least for a small probability, the contribution of input uncertainties in the determination of cascade losses is examined. To this end, $\mathrm{S}_{\mathrm{i}}^{\mathrm{T}}(\mathrm{Y})$ for each optimized cascade are reported in Figure 9. 
Contrary to $\mathrm{S}_{\mathrm{i}}^{\mathrm{T}}(\dot{\mathrm{m}})$, which are equal among the different blades, cascade losses are influenced by input uncertainties in a different way. The first thing to note is that uncertainties in the turbulent boundary conditions and in the inlet flow angle are not inducing any variation in the cascade performance. For such nozzle cascades, turbulence effects have a relevant role in the total-loss coefficient, as clearly discussed in [13] where inviscid and turbulent simulations are compared. Nevertheless, the present analysis suggests that the inlet boundary conditions of the turbulence equations do not have any significant follow-up in the development of turbulent boundary layer on the blade walls, at least according to the RANS model employed in this work. As expected, on the other hand, the large rounded leading edge makes the blade robust to incidence angle up to $\pm 5 \mathrm{deg}$. Since many loss mechanisms are involved, it is not immediate to correlate how the remaining uncertainties, i.e. $\mathrm{T}_{\mathrm{T} 0}, \mathrm{P}_{\mathrm{T} 0}$ and $\mathrm{P}_{1}$, affect the cascade performance within a robust-performance framework.

As a general rule, a deterministic shape optimization applied to a supersonic cascade produces a blade channel which features a smooth and shock-free expansion process. However, the blade is 'adapted' for the design conditions and there is no control about its response to a variation in the boundary conditions. To illustrate a possible physical interpretation of the differences found in $\mathrm{S}_{\mathrm{i}}^{\mathrm{T}}(\mathrm{Y})$, we will consider the two extreme cases: (i) 13CP-DS, where practically the only contribution to losses is given by the total-temperature variation; (ii) 7CP-CNS-DS, where the contribution of the total-temperature uncertainty is smaller than both the inlet total-pressure and the outlet-static-pressure variations. The other cascades are expected to be in between these two exemplary cases. The standard-deviation and the mean pressure fields are combined to illustrate the coefficient of variation $(\mathrm{CoV}=\sigma / \mu)$ fields in Figure 10(a)-(b) for these two representative cascades. Two similar uncertainty peaks are recognized as the shock wave is reflected on the adjacent blade, but a qualitative difference arises when moving downstream. The two peaks are kept separated for the 7CP-CNS-DS, see Figure 10(a), instead they coalesce downstream for the 13CP-DS, see Figure 10(b). This 
difference in the stochastic field proves that a deterministic optimization may grant alternative robust behaviour to the optimized cascade. The azimuthal pressure distribution along with its uncertainty bands (evaluated as twice the standard deviation) are reported for the two cases in Figures $10(c)$ and $10(d)$, respectively. Indeed, the shock pattern generated by the two blades delivers different pressure and uncertainty distribution. Considering the standard deviation of the mean azimuthal-pressure distribution as indicator of the overall change in the downstream flow field, i.e. DP as in Equation 1, the corresponding PDFs are compared in Figure 10(e). 13CP-DS exhibits a downstream pressure field which is more robust to operating uncertainties. On the other hand, the larger variability in the 7CP-CNS-DS PDF indicates a less robust response, as the variation in the operating conditions is likely to trigger a change in shock-wave location and/or intensity.

Although a higher total-temperature contribution seems to indicate a more robust blades in terms of flow-field distribution, a proper physical interpretation, formulated as follows, is required for the generalization. Cascade losses are proportional to the entropy production and to the local temperature at which losses take place, i.e. $\mathrm{Y} \propto \mathrm{T} \Delta \mathrm{S}$ [33]. The overall pressure contributions, given by both the total-upstream and/or the static-downstream pressure, i.e. $\mathrm{S}_{\mathrm{P}_{\mathrm{T} 0}}^{\mathrm{T}}(\mathrm{Y})$ and $\mathrm{S}_{\mathrm{P}_{1}}^{\mathrm{T}}(\mathrm{Y})$, respectively, prevail whenever a slight change in the expansion ratio produces an appreciably different shock interaction and a consequent variation in the entropy generation (proportional to the shock strength). On the other hand, if the total-temperature contribution $\mathrm{S}_{\mathrm{T}_{\mathrm{T}}}^{\mathrm{T}}(\mathrm{Y})$ is predominant, the preserved flow-field uniformity implies a smaller variation in the shock pattern and in the resulting entropy production. Therefore, for the latter blades, cascade losses are mainly influenced by the temperature level rather than by a variation of the shock pattern, inducing overall a more uniform flow field which may be beneficial for the performance of the subsequent cascades. 


\subsection{Impact of objective functions from a UQ perspective}

In this section, blades obtained with the minimization of different objective functions are compared from a probabilistic perspective. In literature, the main dispute for the optimization of a ORC supersonic nozzle cascade is between an entropy-based [20, 15, 22] and a pressure-based $[13,12]$ objective function. Historically, the latter was preferred in inviscid simulations, where the minimization of pressure fluctuations directly acted on the only source of cascade losses, namely the shock loss. When RANS flow solvers are employed, a shift in the trend is observed, preferring an entropy-based objective function which inherently includes viscous phenomena such as shock-boundary layer interaction and shock-induced flow separation at the trailing edge. However, since such a supersonic profile is representative of a first stator, the reduction of pressure fluctuation downstream of the cascade has also the beneficial effect of decreasing the subsequent stator-rotor interactions, with a potential improvement of rotor aerodynamic performance along with a reduction of the aerodynamic forcing. However, whether one objective function brings some additional advantages over the other from a stochastic perspective is still unclear.

In the following analysis, three profiles are considered: 13CP-CNS-DS, 13CP-CNS-DP and 13CP-DP. The latter is included because there is a small probability that it may actually satisfy the constraint. The total-pressure-loss PDFs of the optimized blades and of the baseline are reported in Figure 11 to highlight the effect of a deterministic shape optimization on the resulting profile robustness in terms of overall performance. For all examined cases, the optimization almost halves the cascade losses and improves the robustness of the cascade, whose performance feature a lower variations when subjected to operating uncertainties. All cascade-loss PDFs exhibit nearly the same shape (similar to a normal probability function) with a maximum deviation between the mean and its inferior/superior 0.05-quantiles of $\Delta \mathrm{Y} \approx 0.15 \%$ pts. As far as the unconstrained blade (13CP-DP) is concerned, it always outperforms its constrained counterpart (13CP-CNS-DP); besides, its losses are lower than 
the entropy-based constrained profile (13CP-CNS-DS) with a probability of about $95 \%$. A comparison between the two constrained-optimized profiles shows that the pressure-based profile presents a higher cascade-loss mean value with a lower standard deviation than its entropy-based counterpart. Moreover, despite the higher mean value, there is still a $\approx 40 \%$ of probability that the pressure-based cascade may behave better than the entropy-based one. Based on these results, on the stochastic ground the optimization provides nearly equivalent outcomes regardless the definition of the objective function. Indeed, as the main shock occurring in the baseline cascade significantly contributes both to the overall loss and to the pressure gradients downstream of the cascade, the minimization of the shock strength, with its consequent loss and impact on the boundary-layer development, is pursued by both the objective functions.

A very different trend is appreciated when the flow uniformity downstream of the cascade is of interest, as observed in Figure 12 by DP probability functions. Both objective functions alter the shape of the baseline PDF, inducing a stiffer change in the shock-wave generation for the optimized cascades as operating conditions vary. However, a substantial difference between entropy-based and pressure-based profiles is evident. Despite comparable cascadeloss coefficients, the pressure-based optimized blades feature pressure-gradient PDFs skewed towards low-quantiles, resulting in a more robust response in terms of flow-field distribution. On the contrary, 13CP-CNS-DS presents a nearly-normal distribution centred on a higher value (almost 4 times the pressure-based one). The optimization based on the entropy as objective function do not account for the onset of weak pressure waves (both compression and expansion) downstream of the trailing edge, whose contribution to the entropy production is indeed negligible but they do affect the pressure disuniformity in the stator-rotor gap. In accordance with these findings, the optimization of a ORC supersonic nozzle cascade should involve the minimization of the pressure standard deviation downstream of the trailing edge, even in presence of viscous flow solver, because it results in a twofold advantage: 
(i) minimization of losses, evidencing only a marginal difference in the cascade-loss PDFs;

(ii) reduction of flow disuniformity, outperforming the entropy-based profile from both a deterministic and a stochastic perspectives.

\subsection{Impact of design variables from a UQ perspective}

The last analysis compares the implication of different design spaces in the stochastic responses of the so-optimized profiles. The investigation is carried out by varying the design variables as in Figure 1 and employing the same flow model and optimization set up in terms of objective functions and constraints. The resulting two blades are: 7CP-CNS-DS and 13CP-CNS-DS. It is worth to underline that the 7CP-CNS-DS profile demanded about a half of the computational time, which was required for the optimization of the 13CP-CNSDS. The extra available CPs enable the displacement of the diverging part of the pressure side and a rigid movement of the trailing edge (preserving its thickness). Previous studies $[13,23]$ showed that the improvement in the cascade performance by moving these regions is marginal (but still measurable in deterministic terms), if compared to the one achievable by displacing the diverging and the rear part of the suction side. The cascade-loss PDFs are illustrated in Figure 13 for both the optimized profiles and the baseline. The two PDFs exhibit a similar distribution, which features a lower standard deviation than the baseline one for both the cases. Moreover, it can be noticed that the performance of 7CP-CNS-DS is slightly less robust then its counterpart obtained by a higher CP number. Nevertheless, with reference to the deterministic cascade-loss values, see Table 1, 7CP-CNS-DS outperforms the deterministic performance of 13CP-CNS-DS with a probability of around $25 \%$. Correspondingly, 13CP-CNS-DS might operate worse than the deterministic 7CP-CNS-DS with a probability of $30 \%$. Based on these results, the trailing-edge region and the pressure side downstream of blade throat seem not to provide a significant contribution to the robustness of the blade profile, mirroring the marginal net improvement given by deterministic 
computations [23]. It follows that, within the parametrization framework here employed, the number of adjustable CPs can be restricted to the rear suction side only, thus saving computational resources at nearly equal performance from a stochastic perspective.

\section{CONCLUSION}

In this paper we discuss the impact of a deterministic shape optimization on the performance of supersonic turbine nozzles when operating under uncertainties. The analysis was carried out by resorting to a well-known cascade configuration in the field of Organic Rankine Cycle applications, featuring severe non-ideal gas behaviours and a highly-supersonic flow regime. A turbulent flow solver, relying on a state-of-the-art multi-parameter equation of state, was employed. The uncertainty quantification strategy involved a recently-proposed Polynomial Chaos-Kriging surrogate technique. Within this framework, we tested 5 optimized profiles which were obtained applying deterministic optimizations and changing the objective function (pressure standard deviation - entropy production), the constraint (no constraint mass-flow-rate constraint) and the design space ( 7 - 13 movable control points). The main outcomes from this analysis are summarized as follows:

- the mass-flow-rate probability density function can be deduced a priori for choked supersonic cascades. A 1D-based methodology is proposed to include the mass-flowrate probability density function within the formulation of a deterministic optimization problem, enforcing its robust constraint fulfilment with no extra computational cost;

- overall, a deterministic optimization leads to a more robust design, but the shock-wave pattern and the consequent cascade performance may be affected by uncertainties at least in two different ways;

- uncertainties in the flow boundary conditions have a higher influence on the cascade- 
performance with respect to uncertainties in the turbulent boundary conditions;

- for this kind of turbines, whose losses are dominated by shock waves (and slightly affected by viscous-shock interaction), the best objective function seems to be the standard deviation of the downstream azimuthal pressure distribution. Its minimization increases the flow-field uniformity downstream of the stator, thus eventually improving the stator-rotor interaction, and it has the positive side-effect of reducing cascade losses by removing the main shock;

- the increase in the number of control points, also including the pressure side and the trailing edge, seems not to be justified for the examined nozzle cascades, because the gain in cascade performance is marginal and might be nullified when uncertainties are introduced.

Finally, in the present stochastic context, we found nearly-normal probability density function for cascade-loss coefficients, with a maximum deviation from the mean value of around $\Delta \mathrm{Y} \approx 0.15 \%$ pts. This result leaves small room for further cascade-performance improvements, for example by directly addressing operating uncertainties with much timeconsuming robust-optimization techniques.

An open problem, on the other hand, is how to reduce the cost of the optimization and to preserve accuracy by using lower-fidelity codes, both on the choice of the physical model and on the mesh resolution. In this case, the interest in using an uncertainty-quantification approach to take into account the error introduced by the low-fidelity model still remains to be properly assessed. This problem will be tackled in future works. 


\section{Nomenclature}

$\begin{array}{ll}\text { ORC } & \text { Organic Rankine Cycle } \\ \text { PDF } & \text { Probability Density Function } \\ \text { UQ } & \text { Uncertainty Quantification } \\ \text { DoE } & \text { Design of Experiments } \\ \text { LHS } & \text { Latin Hypercube Sampling } \\ \text { CNS } & \text { Constrained } \\ \text { PCK } & \text { Polynomial Chaos - Kriging } \\ \text { CP } & \text { Control Point } \\ \text { QoI } & \text { Quantity of Interest } \\ \text { ANOVA } & \text { ANalysis Of VAriance }\end{array}$

7CP-CNS-DS Optimal blade obtained with 7 movable CPs, minimizing the entropy production and constraining the mass flow rate

13CP-DS Optimal blade obtained with 13 movable CPs and minimizing the entropy production

13CP-CNS-DS Optimal blade obtained with 13 movable CPs, minimizing the entropy production and constraining the mass flow rate

13CP-DP Optimal blade obtained with 13 movable CPs and minimizing the standard deviation of azimuthal pressure distribution downstream half axial chord of the trailing edge

13CP-CNS-DP Optimal blade obtained with 13 movable CPs, minimizing the standard deviation of azimuthal pressure distribution downstream half axial chord of the trailing edge and constraining the mass flow rate

$\begin{array}{ll}\mathrm{DS} & \text { Specific entropy production (Eq. 1) } \\ \mathrm{DP} & \text { Standard deviation of azimuthal pressure distribution (Eq. 1) } \\ \mathrm{Y} & \text { Total pressure loss coefficient (Eq. 2) } \\ \mathrm{S}^{\mathrm{T}} & \text { Total Sobol index (Eq. 4) } \\ \alpha_{0} & \text { Inlet flow angle } \\ \mathrm{T}_{\mathrm{T} 0} & \text { Inlet total temperature } \\ \mathrm{P}_{\mathrm{T} 0} & \text { Inlet total pressure } \\ \mathrm{P}_{1} & \text { Outlet static pressure } \\ \mathrm{TI} & \text { Turbulence intensity } \\ \mu_{\mathrm{T}} / \mu & \text { Eddy viscosity ratio } \\ \dot{\mathrm{m}} & \text { Mass-flow rate } \\ \mathrm{M} & \text { Mach number } \\ \mathcal{U} & \text { Uniform distribution } \\ \mu & \text { Mean value } \\ \sigma & \text { Standard deviation } \\ \mathrm{CoV} & \text { Coefficient of variation } \sigma / \mu\end{array}$




\section{Tables}

\begin{tabular}{lcccrc}
\hline Blade & $\mathrm{DS}[\mathrm{J} /(\mathrm{kg} \mathrm{K})]$ & $\mathrm{DP}[\mathrm{Pa}]$ & $\mathrm{OF} / \mathrm{OF}_{\text {base }}$ & $\mathrm{Y}[\%]$ & $\dot{\mathrm{m}} / \dot{\mathrm{m}}_{\text {base }}$ \\
\hline \hline BASELINE & 4.46 & 16626 & 1.00 & 17.96 & 1.00 \\
7CP-CNS-DS & 2.31 & 4553 & 0.52 & 9.83 & 1.00 \\
13CP-CNS-DS & 2.29 & 4172 & 0.51 & 9.77 & 1.00 \\
13CP-CNS-DP & 2.32 & 1313 & 0.08 & 9.87 & 1.00 \\
13CP-DS & 2.18 & 5712 & 0.49 & 9.30 & 1.10 \\
13CP-DP & 2.24 & 1156 & 0.07 & 9.54 & 1.03 \\
\hline
\end{tabular}

Table 1: Performance in terms of objective-function reduction, total-pressure loss coefficient and degree of fulfilment of the mass-flow-rate constraint for the baseline and the optimized blade profiles. The results are obtained with $200 \mathrm{k}$ mesh.

\begin{tabular}{ll}
\hline Variable & Range \\
\hline \hline $\mathrm{T}_{\mathrm{T} 0}$ & $\mathcal{U}([541.07,549.23]) \mathrm{K}$ \\
$\mathrm{P}_{\mathrm{T} 0}$ & $\mathcal{U}([7.880,8.120]) \mathrm{bar}$ \\
$\mathrm{P}_{1}$ & $\mathcal{U}([1.061,1.083]) \mathrm{bar}$ \\
$\mathrm{TI}$ & $\mathcal{U}([2.5,7.5]) \%$ \\
$\alpha_{0}$ & $\mathcal{U}([-5,5]) \mathrm{deg}$ \\
$\mu_{\mathrm{t}} / \mu$ & $\mathcal{U}([1,100])-$ \\
\hline
\end{tabular}

Table 2: Uncertain variables and their related range of variation for the following UQ analyses.

\section{Figures}



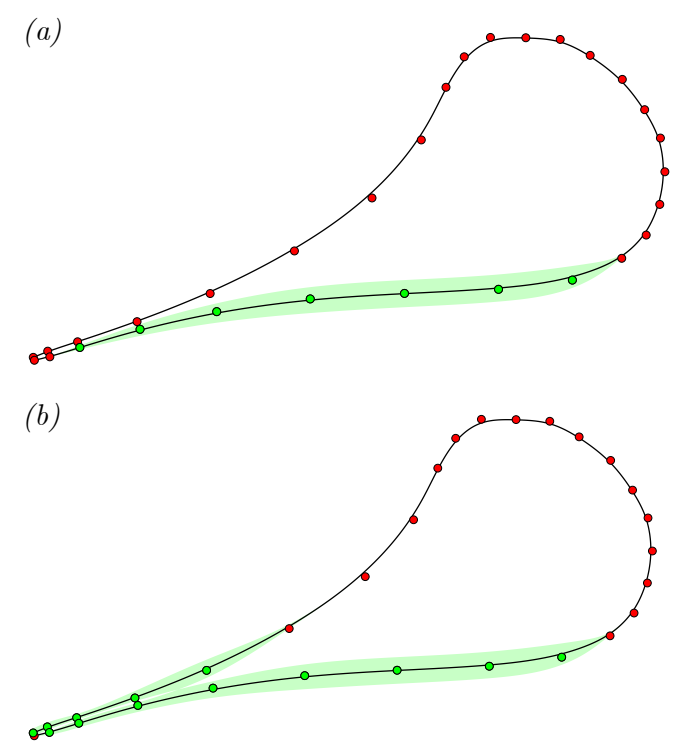

Figure 1: Baseline-cascade parametrization with $7(a)$ and $13(b)$ adjustable control points. The shaded area corresponds to the overall design space.

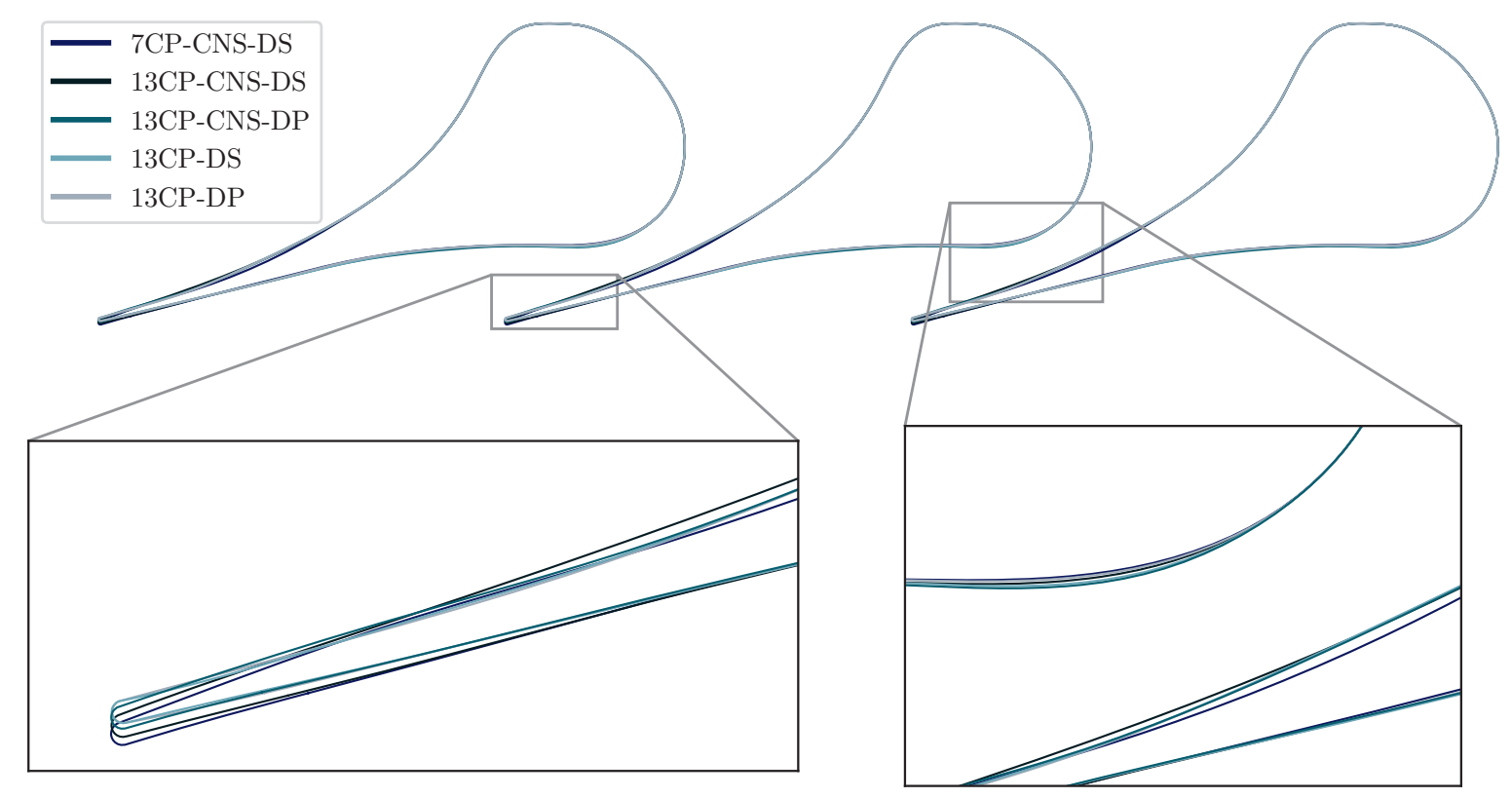

Figure 2: Comparison of optimized blade profiles obtained with different control points (7CP - 13CP), objective functions (DS: entropy production - DP: standard deviation of azimuthal pressure distribution) and constraints (CNS: mass flow constrained within $\pm 1 \%$ with respect to baseline value). 

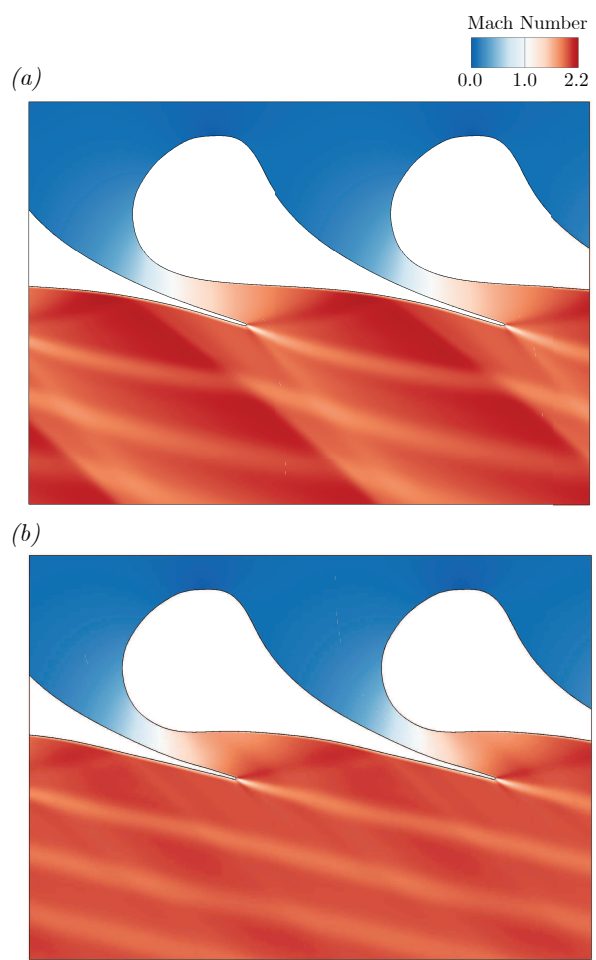

Figure 3: Mach number flow field of the baseline cascade $(a)$ and of the optimized blade 13CP-DS $(b)$. The numerical simulations are performed with 200k mesh.
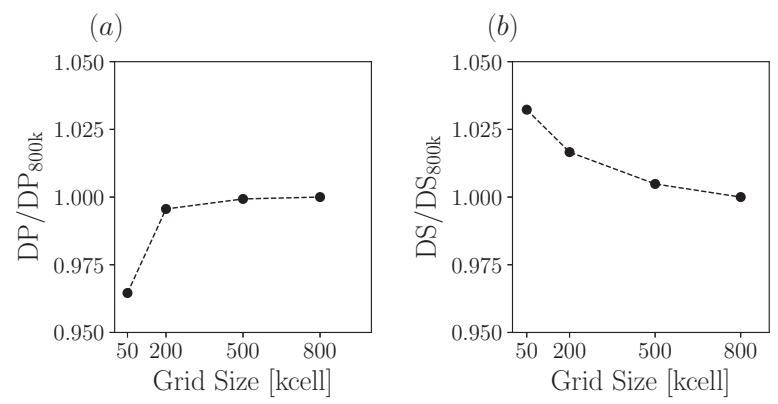

Figure 4: Grid-convergence assessment for the baseline cascades in terms of standard deviation of azimuthal pressure distribution $(a)$ and entropy production $(b)$, normalized to the finest-grid value. 


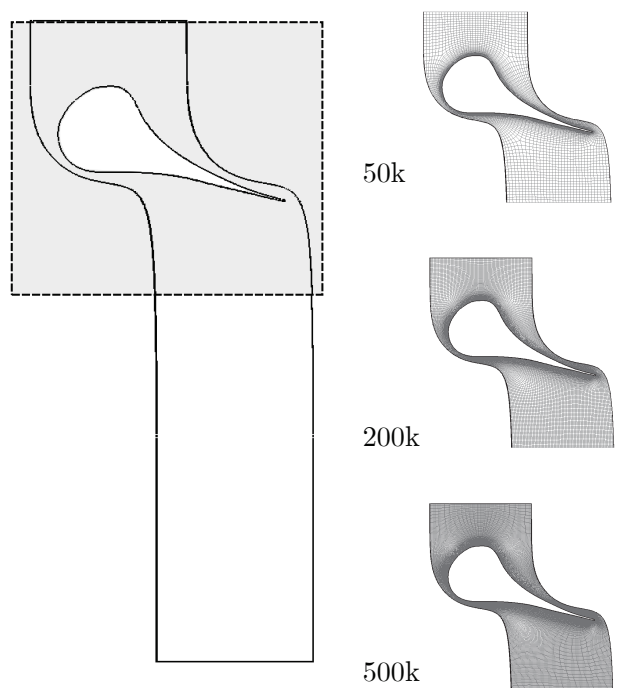

Figure 5: Mesh refinements for the stochastic grid-convergence assessment. The medium mesh (200k) showed the best compromise between accuracy and computational cost. 


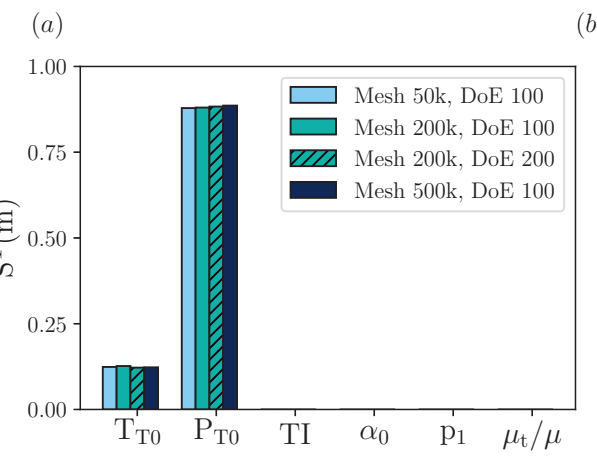

(b)
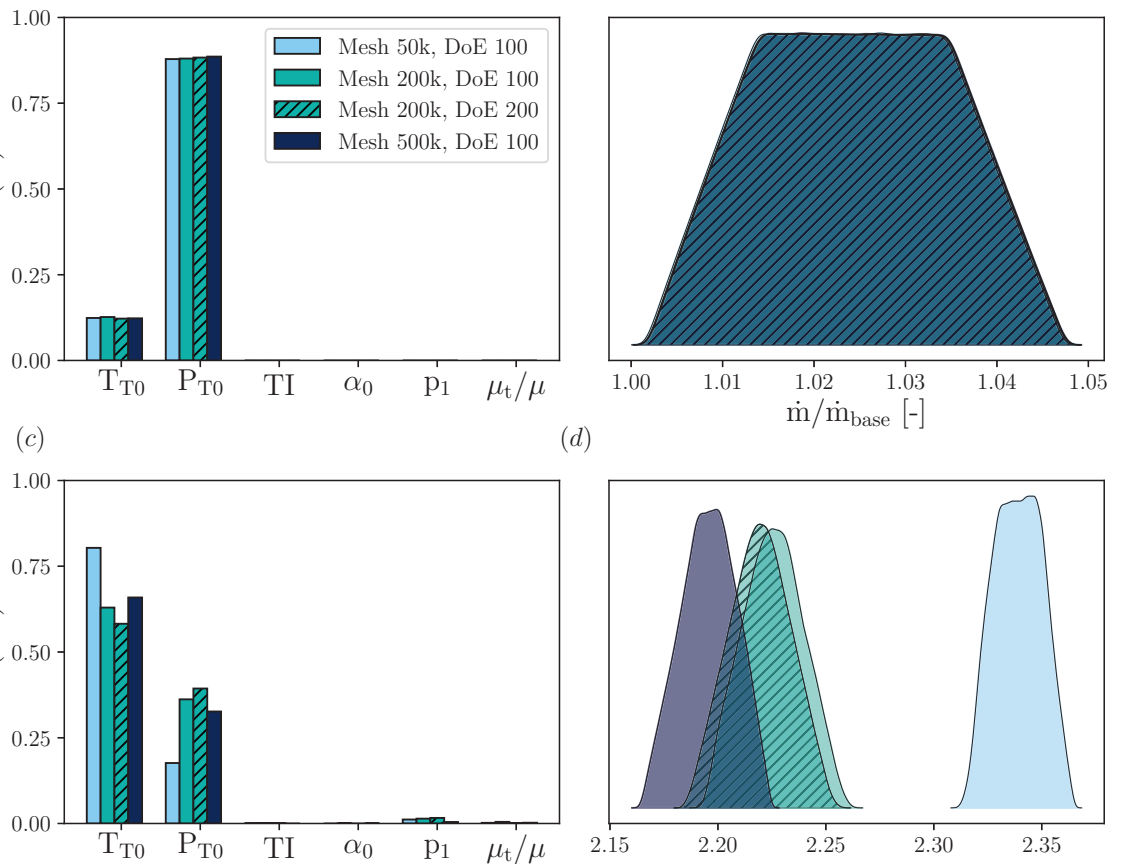

$(d)$

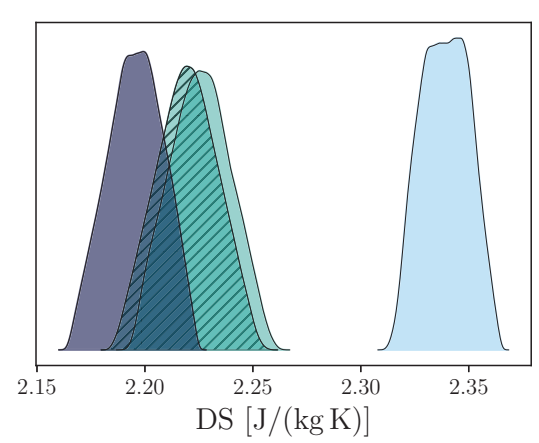

Figure 6: Evolution of sensitivity indexes and of PDFs in terms of mesh size for CFD evaluation and size of Design of Experiments (DoE) to tune the PCK surrogate. The analysis is performed on the representative optimized profile 13CP-DP. (a) total Sobol indices of the mass-flow rate; (b) PDFs of the mass-flow rate; $(c)$ total Sobol indices of the entropy production; $(d)$ PDFs of the entropy production. 


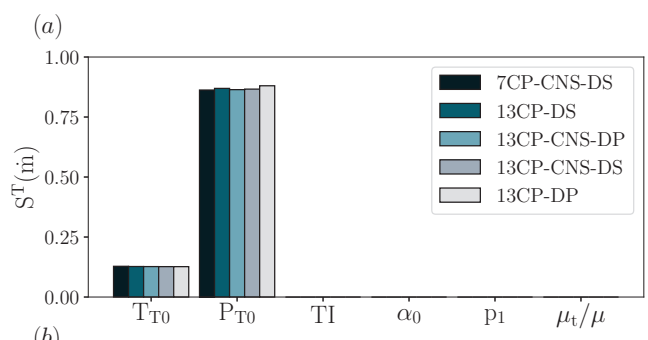

(b)

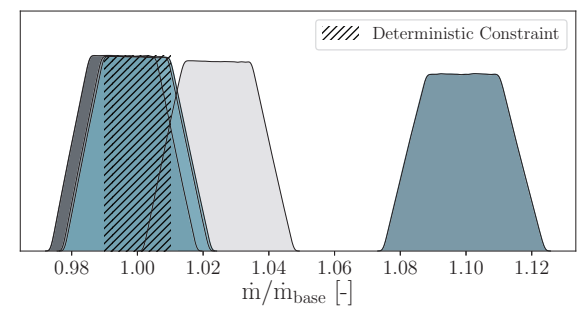

Figure 7: Total Sobol indices $(a)$ and PDFs $(b)$ of the mass-flow rate for the optimized blade profiles. The area filled with solid lines indicates the acceptable degree of unfulfilment from the penalty formulation in the present deterministic optimizations.

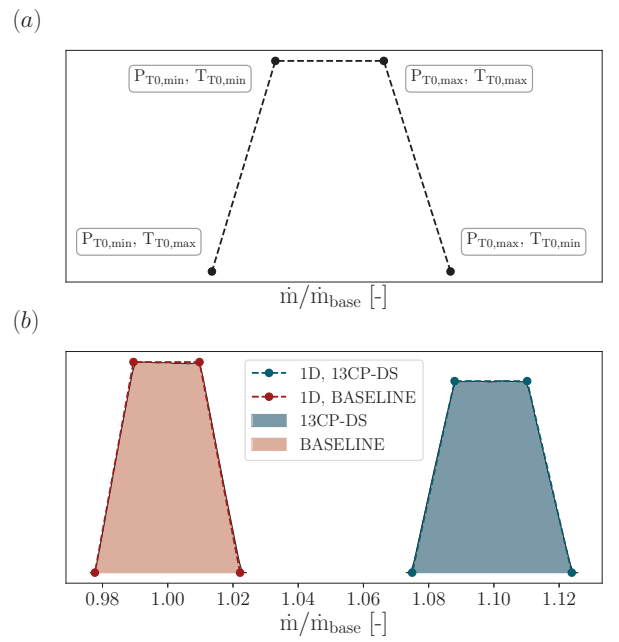

Figure 8: (a) Schematic construction of the mass-flow-rate PDF using the proposed 1D analytical approach; (b) Comparison between analytically-generated (dashed lines with markers) and UQ-generated (shaded area) mass-flow-rate PDFs. 


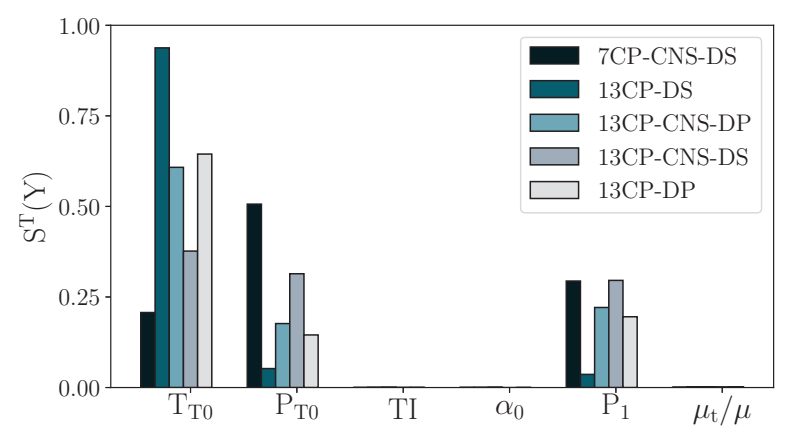

Figure 9: Total Sobol indices of the total-pressure loss coefficient for the optimized blade profiles.

(a)

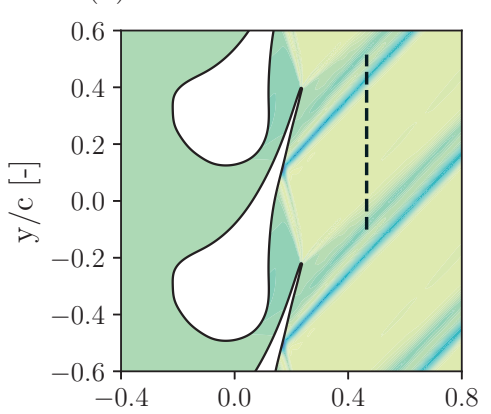

(b)

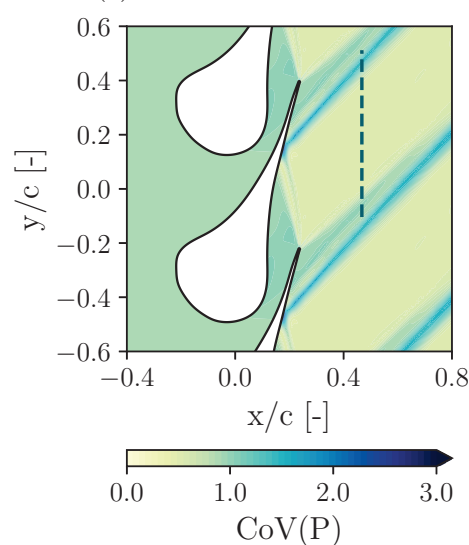

(c)

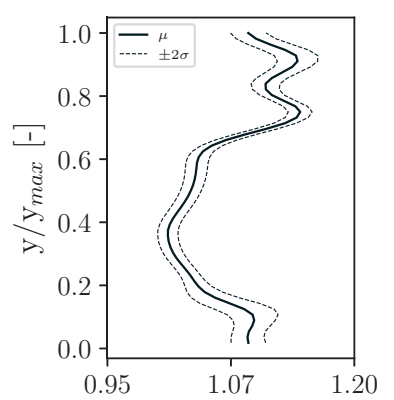

(d)

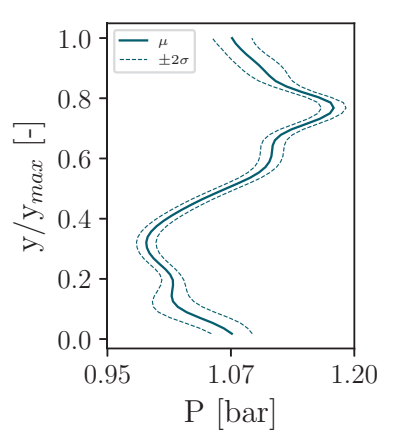

(e)

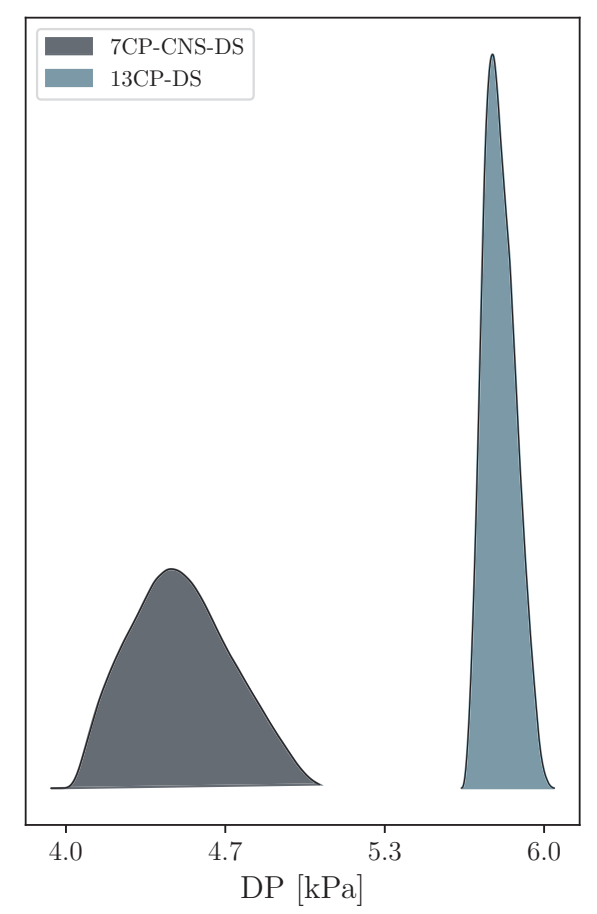

Figure 10: Coefficient-of-Variation contours of the pressure field for 7CP-CNS-DS $(a)$ and 13CP-DS $(b)$ profiles. Dotted lines indicates where azimuthal pressure distributions are evaluated, as reported along with their uncertainty bands in $(c)$ and $(d)$, respectively. The corresponding PDFs of azimuthal-pressure-distribution standard deviation are reported in $(e)$. 

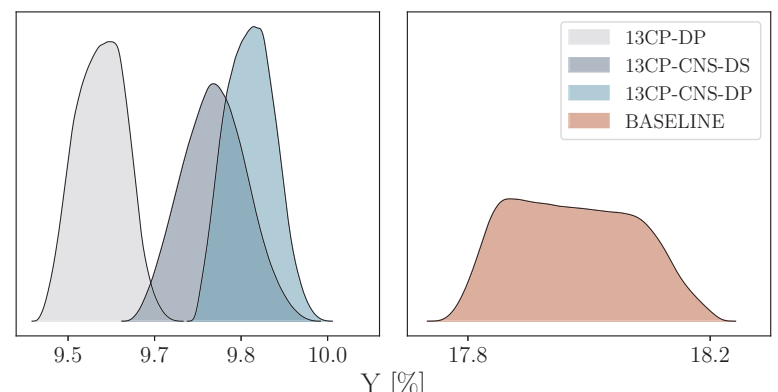

Figure 11: Comparison of total-pressure loss coefficient PDFs for the blade profiles optimized with different objective functions.
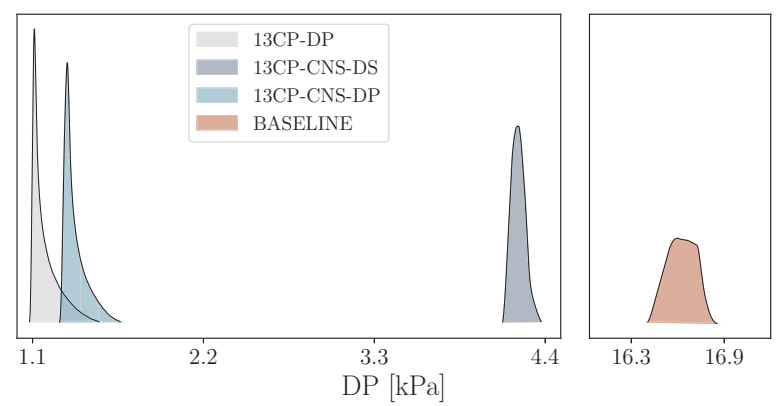

Figure 12: Comparison of azimuthal-pressure-distribution standard deviation PDFs for the blade profiles optimized with different objective function.

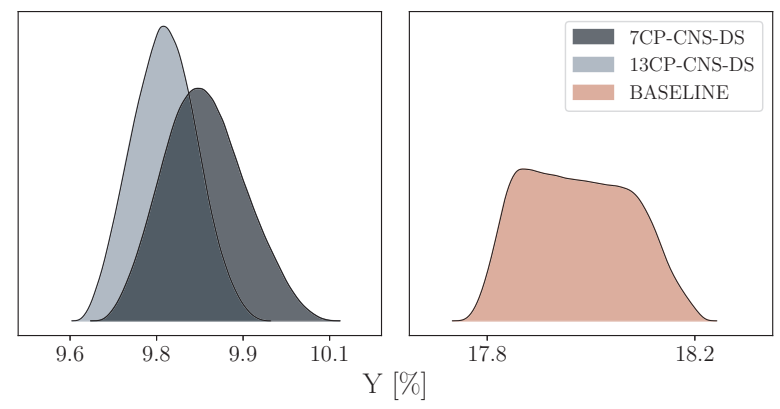

Figure 13: Comparison of total-pressure loss coefficient PDFs for the blade profiles optimized with different control points. 


\section{References}

[1] P. Colonna, E. Casati, C. Trapp, T. Mathijssen, J. Larjola, T. Turunen-Saaresti, and A. Uusitalo, "Organic Rankine Cycle Power Systems: From the Concept to Current Technology, Applications, and an Outlook to the Future," Journal of Engineering for Gas Turbines and Power, vol. 137(10), pp. 1-10, 2015.

[2] E. Macchi, "1 -Theoretical basis of the Organic Rankine Cycle," in Organic Rankine Cycle (ORC) Power Systems (E. Macchi and M. Astolfi, eds.), pp. 3-24, Woodhead Publishing, 2017.

[3] P. Colonna, J. Harinck, S. Rebay, and A. Guardone, "Real-Gas Effects in Organic Rankine Cycle Turbine Nozzles," J. Propul. Power, vol. 24, no. 2, pp. 282-294, 2008.

[4] E. Macchi and M. Astolfi, "9 - Axial flow turbines for Organic Rankine Cycle applications," in Organic Rankine Cycle (ORC) Power Systems (E. Macchi and M. Astolfi, eds.), pp. 299-319, Woodhead Publishing, 2017.

[5] J. Hoffren, T. Talonpoika, J. Larjola, and T. Siikonen, "Numerical simulation of real-gas flow in a supersonic turbine nozzle ring," Journal of Engineering for Gas Turbines and Power, vol. 124, pp. 395-403, 2002.

[6] J. Harinck, D. Pasquale, R. Pecnik, J. van Buijtenen, and P. Colonna, "Performance improvement of a radial Organic Rankine Cycle turbine by means of automated computational fluid dynamic design," Proceedings of the Institution of Mechanical Engineers, Part A: Journal of Power and Energy, vol. 227, no. 6, pp. 637-645, 2013.

[7] G. Persico and M. Pini, "8 - Fluid dynamic design of Organic Rankine Cycle turbines," in Organic Rankine Cycle (ORC) Power Systems (E. Macchi and M. Astolfi, eds.), pp. 253 - 297, Woodhead Publishing, 2017. 
[8] E. Rinaldi, R. Pecnik, and P. Colonna, "Unsteady Operation of a Highly Supersonic ORC Turbine," Journal of Turbomachinery, vol. 128, pp. 1-9, 2016.

[9] F. Rubechini, M. Marconcini, A. Arnone, A. S. Del Greco, and R. Biagi, "Special challenges in the computational fluid dynamics modeling of transonic turbo-expanders," Journal of Engineering for Gas Turbines and Power, vol. 135, 2013.

[10] E. A. Bufi and P. Cinnella, "Preliminary design method for dense-gas supersonic axial turbine stages," Journal of Engineering for Gas Turbines and Power, vol. 140, pp. 1-11, 2018.

[11] N. Anand, S. Vitale, M. Pini, G. J. Otero, and R. Pecnik, "Design methodology for supersonic radial vanes operating in non-ideal flow conditions," Journal of Engineering for Gas Turbines and Power, vol. 141, pp. 1-9, 2018.

[12] D. Pasquale, A. Ghidoni, and S. Rebay, "Shape optimization of an Organic Rankine Cycle radial turbine nozzle," Journal of Engineering for Gas Turbines and Power, vol. 135, pp. 1-13, 2013.

[13] M. Pini, G. Persico, D. Pasquale, and S. Rebay, "Adjoint Method for Shape Optimization in Real-Gas Flow Applications," Journal of Engineering for Gas Turbines and Power, vol. 137, pp. 1-13, 2015.

[14] P. R. Fernandez and G. Persico, "Automatic design of ORC turbine profiles using evolutionary algorithms," 3rd International Seminar on ORC Power System (ORC2015), Brussels, Belgium, Oct 12-14, 2015.

[15] S. Vitale, T. A. Albring, M. Pini, N. R. Gauger, and P. Colonna, "Fully turbulent discrete adjoint solver for non-ideal compressible flow applications," Journal of the Global Power and Propulsion Society, vol. 1, pp. 252-270, 2017. 
[16] H.-G. Beyer and B. Sendhoff, "Robust optimization - a comprehensive survey," Computer Methods in Applied Mechanics and Engineering, vol. 196, pp. 3190-3218, 2007.

[17] P. M. Congedo, C. Corre, and J. M. Martinez, "Shape Optimization of an airfoil in a BZT flow with multiple-source uncertainties," Comput. Methods Appl Mech. Engrg., no. 200, pp. 216-232, 2011.

[18] P. M. Congedo, G. Geraci, R. Abgrall, V. Pediroda, and L. Parussini, "TSI metamodelsbased multi-objective robust optimization," Engineering Computations, vol. 30, no. 8, pp. 1032-1053, 2013.

[19] E. A. Bufi, P. Cinnella, and X. Merle, "Sensitivity of Supersonic ORC Turbine Injector Designs to Fluctuating Operating Conditions," ASME Turbo Expo: Turbomachinery Technical Conference and Exposition, Montreal, Canada, June 15-19, 2015.

[20] E. A. Bufi and P. Cinnella, "Robust optimization of supersonic ORC nozzle guide vanes," Journal of Physics: Conference Series, vol. 821, pp. 1-10, 2017.

[21] L. Zanellato, M. Astolfi, A. Serafino, D. Rizzi, and E. Macchi, "Field Performance Evaluation of ORC Geothermal Power Plants Using Radial Outflow Turbines," Energy Procedia, vol. 129, pp. $607-614,2017$.

[22] G. Persico, "Evolutionary Optimization of Centrifugal Nozzles for Organic Vapours," Journal of Physics: Conference Series, vol. 821, 2017.

[23] A. Romei and G. Persico, "Novel Shape Parametrization Technique applied to the Optimization of a Supersonic ORC Turbine Cascade," ASME Turbo Expo: Turbomachinery Technical Conference and Exposition, Oslo, Norway, June 11-15, 2018.

[24] M. Thol, F. H. Dubberke, E. Baumhögger, J. Vrabec, and R. Span, "Speed of Sound Measurements and Fundamental Equations of State for Octamethyltrisiloxane and 
Decamethyltetrasiloxane," Journal of Chemical \& Engineering Data, vol. 62, no. 9, pp. 2633-2648, 2017.

[25] E. W. Lemmon, M. L. Huber, and M. O. McLinden, "NIST reference database 23: reference fluid thermodynamic and transport properties-REFPROP, version 9.1," Standard Reference Data Program, 2013.

[26] G. Persico, A. Mora, P. Gaetani, and M. Savini, "Unsteady Aerodynamics of a Low Aspect Ratio Turbine Stage: Modeling Issues and Flow Physics," Journal of Turbomachinery, vol. 134, no. 1, pp. 1 - 10, 2012.

[27] P. Kersaudy, B. Sudret, N. Varsier, O. Picon, and J. Wiart, "A new surrogate modeling technique combining Kriging and Polynomial Chaos Expansions - Application to uncertainty analysis in computational dosimetry," Journal of Computational Physics, vol. 286, pp. 103-117, 2015.

[28] C. Rasmussen and C. Williams, "Gaussian processes for machine learning," Adaptive computation and machine learning, Cambridge, Massachusetts: MIT Press, 2006.

[29] G. Blatman and B. Sudret, "Adaptive sparse polynomial chaos expansion based on Least Angle Regression," Journal of Computational Physics, vol. 230, pp. 2345-2367, 2011.

[30] A. Janon, T. Klein, A. Lagnoux, M. Nodet, and C. Prieur, "Asymptotic normality and efficiency of two sobol index estimators," ESAIM: Probability and Statistics, vol. 18, pp. 342-364, 2014.

[31] B. Sudret and S. Marelli, "UQLab - The Framework for Uncertainty Quantification, version 1," http://www.uqlab.com/ (19-07-2018). 
[32] B. W. Silverman, "Density Estimation for Statistics and Data Analysis," Chapman and Hall/CRC Monographs on Statistics and Applied Probability, 1986.

[33] J. D. Denton, "Loss mechanisms in turbomachines," Journal of Turbomachinery, vol. 115, pp. 621-651, 1993. 